\title{
Is PSMA PET-CT Better than Bone Scan? When and Why
}

\author{
Manoj Gupta ${ }^{1^{*}}$, Partha Sarathi Choudhury ${ }^{1}$, Harish Chandra Goel ${ }^{2}$, Sudhir Rawal ${ }^{3}$ and Vineet Talwar ${ }^{4}$
}

${ }^{1}$ Department of Nuclear Medicine, Rajiv Gandhi Cancer Institute and Research Centre, New Delhi, India

${ }^{2}$ Amity centre for radiation biology, Amity University, Noida, Uttar Pradesh, India

${ }^{3}$ Department of Uro-Gynae Surgical Oncology, Rajiv Gandhi Cancer Institute and Research Centre, New Delhi, India

${ }^{4}$ Department of Medical Oncology, Rajiv Gandhi Cancer Institute and Research Centre, New Delhi, India

*Corresponding author: Manoj Gupta, Department of Nuclear Medicine, Rajiv Gandhi Cancer Institute and Research Centre, New Delhi, India, Tel: 011 4702 2222; Email: docmanojgupta@yahoo.com

Received date: October 20, 2017; Accepted date: October 25, 2017; Published date: November 01, 2017

Copyright: () 2017 Gupta M, et al. This is an open-access article distributed under the terms of the Creative Commons Attribution License, which permits unrestricted use, distribution, and reproduction in any medium, provided the original author and source are credited.

\begin{abstract}
Prostate cancer $(\mathrm{PCa})$ has a unique tropism to bone. Indeed, bone is the most frequent site of distant metastasis and cause of morbidity due to skeletal complications. 99mTc-Methylene diphosphonate (MDP) bone scintigraphy/ scan (BS) is the current standard imaging due to increase adsorption of the tracer at osteoblastic sites. However, it has limited specificity due to false positives in degenerative changes, benign causes and false negatives in bone marrow metastasis and lytic lesions. Another drawback of BS is flare response. Prostate Specific Membrane Antigen (PSMA) has been the most studies target in prostate cancer imaging in recent time due to 100-1000 time overexpression in cancer cells. 68Ga-PSMA-11, a small molecule with PSMA enzyme inhibition activity has been found promising in recurrence and lymph-node staging. In our experience of 97 staging prostate cancer patients, PSMA PET-CT showed $57.41 \%$ with pure sclerotic metastasis. Mixed $(33.33 \%)$, marrow $(7.14 \%)$ and lytic $(2.3 \%)$ types of lesions constitute the rest and thus BS alone in these patients may leads to underestimation of bony disease burden. PSMA has not been found positive in degenerative changes however its role in response to anti-androgen needs caution due to know synergistic effect on PSMA expression. We concluded, PSMA PET-CT would have better sensitivity and specificity due to unique distinction for detecting non-sclerotic metastases. We presumed if PSMA has been performed for staging workup then there is limited role of BS except in clinical trial patient. Overall PSMA PET may become one-stop-shop for PCa workup.
\end{abstract}

Keywords: PSMA PET-CT; Bone scan; Marrow metastasis; Lytic metastasis; One-stop-shop

\section{Introduction}

Prostate cancer $(\mathrm{PCa})$ is the second most common cancer and sixth leading cause of cancer death in man worldwide [1]. In India though the incidence is less than the western world, it is showing a rising trend now. Indeed in many metro-cities like Delhi it has become the runners up with age-adjusted incidence of $10.9 / 10^{5}$ person-years [2]. A large number of patients diagnosed with early stage PCa got cured with definitive local therapy i.e. Radical prostatectomy or Radiotherapy, however many will develop metastatic disease. PCa has a unique exquisite tropism to spread in bone [3]. Haematogenous spread in red bone marrow of axial and proximal appendicular skeleton leads to development of bone metastases (BMs). BMs are the most frequent and main distant metastatic site in about $80 \%$ of PCs patients and is therefore one of the most important determinants of treatment and outcome [4,5]. Skeletal complications known as 'skeletal-related events (SREs)' accounts for most of the PCa's morbidity and mortality [6]. Bone marrow replacement by PCa cells leads to anaemia while involvement of cortical bone can lead to pain, fractures, and spinal cord compression. Once bone metastasis is diagnosed, local definitive treatment goes out of the picture and the intent of treatment become palliative. Hence timely diagnosis of bone metastasis is important for correct treatment planning and prevention of SREs.

Bone scintigraphy/scan (BS) with 99mTc-Methylene diphosphonate (MDP) is the most favoured investigation for detecting BMs. This is due to physiological adsorption of this radiopharmaceutical at the site of osteoblastic activity. In PCa BMs there is predominant upregulation of osteoblasts lead to formation of characteristic sclerotic lesions. Hence this method has high sensitivity (range 62-89\%) for BMs in PCa [7]. Briganti has showed risk on BMs in low (Gleason $\leq 7, \mathrm{~T} 2-\mathrm{T} 3$ and $\mathrm{PSA}<10 \mathrm{ng} / \mathrm{ml}$ ), intermediate (Gleason $\leq 7, \mathrm{~T} 2-\mathrm{T} 3$ and PSA $>10 \mathrm{ng} / \mathrm{ml}$ ) and high risk (Gleason $>7$ ) PCa of $1.8 \%, 8.5 \%$ and $16.4 \%$ respectively [8]. Therefore, most guidelines suggest BS to be performed in patients with high risk PCa or those presenting with bone symptoms [9-11].

BS has been associated with number of limitations as well. It is a well-known fact that BM begins in bone marrow, hence it is predicted that BS will not able to detect bone marrow lesions or early lesion with insufficient osteoblastic activity. In addition it is a non-specific tracer and many a times it is hard to differentiate between degenerative bone disease and $\mathrm{BMs}$ hence frequently requiring additional imaging modality for characterization [12]. With modern hybrid imaging SPECT-CT (Single Photon Emission Computed TomographyComputed Tomography), MDP BS has largely addressed this issue of low specificity and able to correctly characterized planner imaging equivocal lesions. It has been reported that the number of equivocal lesions dropped from 61 to $8 \%$ with addition of SPECT-CT [13]. Flare response is another known fact in BS [14]. Post treatment increase in tracer activity or new lesion is tricky in interpretation. Whether this is due to reparative response or due to disease progression is a matter of concern. Nonetheless this phenomenon has been assumed as response by most physicians and presumed to have better outcome. 
Despite these limitations, bone scan has been recommended as standard for BMs in clinical trials by prostate cancer working group. Reason being it is widely available, low cost, time tested and whole body imaging. In addition it has been reported superior to X-Ray and CT [15], roughly equivalent to 11C-Choline-positron emission tomography (PET) [16] as well. Though it is inferior to whole body MRI [17] and 18F-Fluoride PET [18] but these imaging has still not able to find their way in clinical practice and associated with few limitations as well.

Recently prostate-specific membrane antigen (PSMA) has been acclaimed as a distinct target in PCa. Its expression is 100-1000 times more in PCa cells [19] and level of expression is directly proportional to gleason score, androgen independence, metastasis and progression [20]. Many monoclonal antibodies and small molecule inhibitors have been developed to target PSMA. Out of these, a small molecule inhibitor Glu-NH-CO-NH-Lys-(Axe)-[68Ga(HBED-CC)] (68GaPSMA-11) is being most investigated. It has shown to be of high clinical value for lymph node staging [21] and detection of local recurrence $[22,23]$. For BMs PSMA PET has unique distinction of being positive in bone marrow metastasis and not being positive in degenerative bone disease. In a direct comparison, PSMA PET outperformed planner BS for detection of affected bone regions as well as overall bone disease volume $[24,25]$. Overall $17.6 \%$ of affected bone regions were exclusively recognized only by PSMA PET while only $1.2 \%$ of bony regions exclusively detected by BS. PSMA-PET showed significantly higher sensitivity and accuracy than BS $(90.5 \%$ vs. 73.68\%, and $97.0 \%$ vs. 86\%) for BMs [26]. In our experience of 97 staging PSMA PET studies, we found only $57.41 \%$ of patients with BMs had pure sclerotic lesions. Mixed (33.33\%), marrow (7.14\%) and lytic $(2.3 \%)$ types of lesions constitute the rest and thus BS alone in these patients may leads to underestimation of bony disease burden. We found that overall the PSMA PET allows envisioning an all-in-one metastatic work up (both visceral and bone) in high risk prostate cancer (Figures 1 and 2). In addition, we believe PSMA PET will have upper hand in response evaluation of BMs then BS however, data is deficient in the literature.

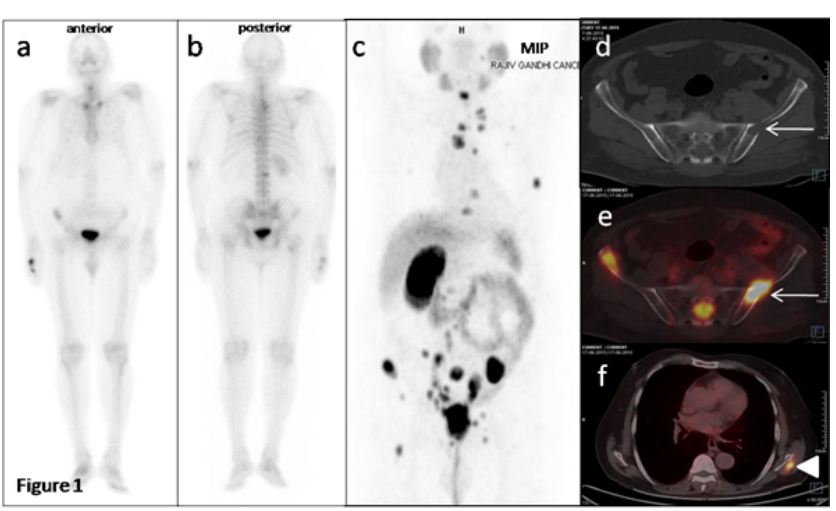

Figure 1: 68 Years male with adenocarcinoma prostate, gleason score $4+5$, PSA $121.5 \mathrm{ng} / \mathrm{ml}$ underwent MDP bone scan (image a and $\mathrm{b}$ ) and 68GaPSMA PET-CT (image $\mathrm{c}, \mathrm{d}, \mathrm{e}, \mathrm{f}$ ). MDP bone scan shows doubtful lesions in right iliac crest, D3 and L2 vertebrae. PSMA PET-CT showed locally infiltrating prostate lesion with pelvic lymphnodes, multiple osteolytic bony lesions (arrow) and a left infraspinatus muscle deposit (arrow head).

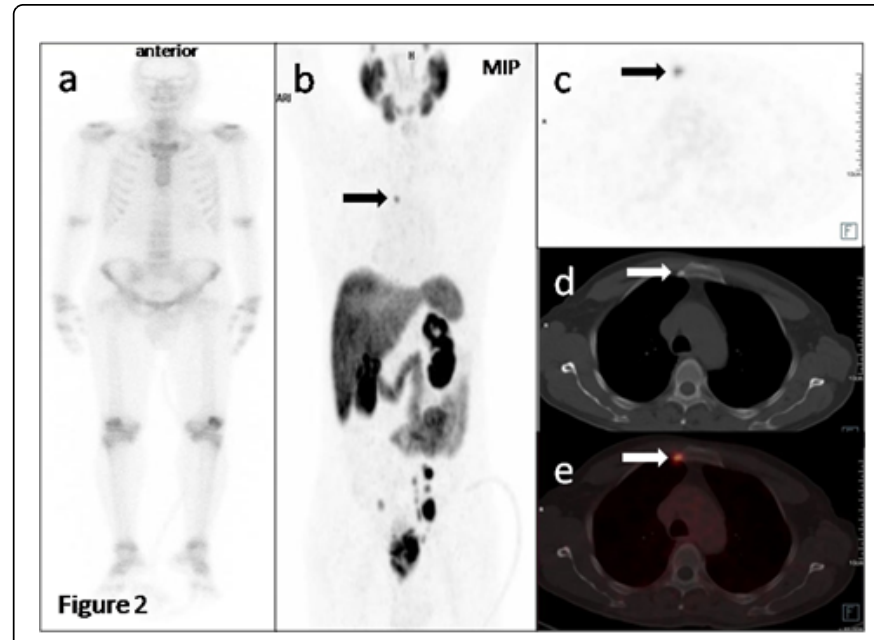

Figure 2: 62 Years male with adenocarcinoma prostate, gleason score $5+4$, PSA $17.5 \mathrm{ng} / \mathrm{ml}$ underwent MDP bone scan (image a) and 68GaPSMA PET-CT (image b, c, d, e). MDP bone scan was reported normal while PSMA PET-CT showed locally infiltrating prostate lesion with pelvic lymphnodes and a solitary bony lesion in sternum (block arrows).

Nevertheless we need to understand that PSMA PET is still in infancy stage and no prospective data is available for its role in BMs. Availability limited to few tertiary care cancer institutes is a big challenge for PSMA PET to come in main stream. Cost and reimbursement are other critical points here for PSMA PET as BS is often covered in health insurance. With growing availability of SPECTCT makes BS specific and a strong contender to PSMA PET in BMs especially in advanced diseases. It has been noticed that PSMA expression is inversely related to androgens level hence, its expression will increase in androgen deprivation state [27]. This influence of anti androgens on PSMA expression is requiring attention in interpretation of response as initial flare up to 3 months can be expected [28,29]. Further studies might be interested in order to disentangle this treatment dependency of PSMA in response assessment of BMs.

\section{Conclusion}

We concluded PSMA PET has better sensitivity and specificity then BS and a unique distinction for detecting non-sclerotic metastases. Its role in response evaluation to anti-androgens needs caution and further studies. We presumed if PSMA has been performed for staging workup then there is limited role of BS except in clinical trial patient. Overall PSMA PET may become one-stop-shop for PCa workup.

\section{References}

1. Jain S, Saxena S, Kumar A (2014) Epidemiology of prostate cancer in India. Meta Gene 2: 596-605.

2. Hariharan K, Padmanabha V (2016) Demography and disease characteristics of prostate cancer in India. Indian J Urol. 32: 103-108.

3. Jin JK, Dayyani F, Gallick GE (2011) Steps in Prostate Cancer Progression that lead to Bone Metastasis. Int J Cancer 128: 2545-2561.

4. Rigaud J, Tiguert R, Le Normand L, Karam G, Glemain P, et al. (2002) Prognostic value of bone scan in patients with metastatic prostate cancer treated initially with androgen deprivation therapy. J Urol 168: 1423-1426. 
5. Soloway MS, Hardeman SW, Hickey D, Raymond J, Todd B, et al. (1988) Stratification of patients with metastatic prostate cancer based on extent of disease on initial bone scan. Cancer 61: 195-202.

6. Norgaard M, Jensen AO, Jacobsen JB, Cetin K, Fryzek JP, et al. (2010) Skeletal related events, bone metastasis and survival of prostate cancer: A population based cohort study in Denmark (1999 to 2007). J Urol 184: 162-167.

7. Daldrup-Link HE, Franzius C, Link TM, Laukamp D, Sciuk J, et al. (2001) Whole-body MR imaging for detection of bone metastases in children and young adults: Comparison with skeletal scintigraphy and FDG PET. Am J Roentgenol 177: 229-236.

8. Briganti A, Passoni N, Ferrari M, Salonia A, Messa C, et al. (2010) When to perform bone scan in patients with newly diagnosed prostate cancer External validation of the currently available guidelines and proposal of a novel risk stratification tool. Eur Urol 57: 551-558.

9. Cook GJ, Azad G, Padhani AR (2016) Bone imaging in prostate cancer: The evolving roles of nuclear medicine and radiology. Clin Transl Imaging 4: 439-447.

10. Wollin DA, Makarov DV (2015) Guideline of guidelines: Imaging of localized prostate cancer. BJU Int 116: 526-530.

11. Heidenreich A, Bastian PJ, Bellmunt J, Bolla M, Joniau S, et al. (2014) EAU guidelines on prostate cancer. part 1: Screening, diagnosis, and local treatment with curative intent-update 2013. Eur Urol 65: 124 -137.

12. Messiou C, Cook G, deSouza NM (2009) Imaging metastatic bone disease from carcinoma of the prostate. Br J Cancer 101: 1225-1232.

13. Helyar V, Mohan HK, Barwick T, Clarke SE, Fogelman I, et al. (2010) The added value of multislice SPECT/CT in patients with equivocal bony metastasis from carcinoma of the prostate. Eur J Nucl Med Mol Imaging 37: 706-713.

14. Cook GJ, Venkitaraman R, Sohaib AS, Chua SC, Parker CC, et al. (2011) The diagnostic utility of the flare phenomenon on bone scintigraphy in staging prostate cancer. Eur J Nucl Med Mol Imaging 38: 7-13.

15. Lee N, Fawaaz R, Olsson CA, Benson MC, Singh A, et al. (2000) Which patients with newly diagnosed prostate cancer need a radionuclide bone scan? an analysis based on 631 patients. Int J Radiat Oncol Biol Phys 48: 1443-1446.

16. Picchio M, Spinapolice EG, Fallanca F, Messa C, Gianolli L, et al. (2012) [11C]Choline PET/CT detection of bone metastases in patients with PSA progression after primary treatment for prostate cancer: Comparison with bone scintigraphy. Eur J Nucl Med Mol Imaging 39: 13-26.

17. Lecouvet FE, El Mouedden J, Collette L, Coche E, Tombal B, et al. (2012) Can whole-body magnetic resonance imaging with diffusion weighted imaging replace Tc $99 \mathrm{~m}$ bone scanning and computed tomography for single-step detection of metastases in patients with high-risk prostate cancer? Eur Urol 62: 68-75.
18. Even-Sapir E, Metser U, Mishani E, Lerman H, Lievssitz G, et al. (2006) The detection of bone metastases in patients with high-risk prostate cancer: 99mTc-MDP Planar bone scintigraphy, single- and multi-field-ofview SPECT, 18F-fluoride PET, and 18F-fluoride PET/CT. J Nucl Med 47: 287-97.

19. Silver DA, Pellicer I, Fair WR, Heston WD, Cordon-Cardo C (1997) Prostate-specific membrane antigen expression in normal and malignant human tissues. Clin Cancer Res 3: 81-5.

20. Chang SS (2004) Overview of prostate-specific membrane antigen. Rev Urol 6: S13-S18.

21. Gupta M, Choudhury PS, Hazarika D, Rawal S (2017) A Comparative study of 68Gallium-prostate Specific membrane antigen positron emission tomography-computed tomography and magnetic resonance imaging for lymph node staging in high risk prostate cancer patients: An initial experience. World J Nucl Med 16: 186-191.

22. Eiber M, Maurer T, Souvatzoglou M, Beer AJ, Wester HJ, et al.(2015) Evaluation of Hybrid 68Ga-PSMA Ligand PET/CT in 248 Patients with Biochemical Recurrence After Radical Prostatectomy. J Nucl Med 56: 668-674.

23. Perera M, Papa N, Christidis D, Hofman MS, Bolton D, et al. (2016) Sensitivity, specificity, and predictors of positive $68 \mathrm{Ga}$-prostate-specific membrane antigen positron emission tomography in advanced prostate cancer: A systematic review and meta-analysis. Eur Urol 70: 926-937.

24. Pyka T, Okamoto S, Dahlbender M, Heck M, Eiber M, et al. (2016) Comparison of bone scintigraphy and 68Ga-PSMA PET for skeletal staging in prostate cancer. Eur J Nucl Med Mol Imaging 43: 2114-2121.

25. Schreiter V, Gericke M, Heimann U, Steffen I, Hamm B, et al. (2016) Comparison of [68Ga]Ga-PSMA-HBED-CC PET versus whole-body bone scintigraphy for the detection of bone metastases in patients with prostate cancer. J Nucl Med Radiat Ther 7: 302.

26. Lengana T, Modiselle M, Lawal I, Ebenhan T, Vorster M, et al. (2017) 68Ga-PSMA-PET/CT and bone scintigraphy imaging for staging of highrisk prostate cancer. J Nucl Med 58: 757.

27. Wright GL Jr, Grob BM, Haley C, Newhall K, Troyer J, et al. (1996) Upregulation of prostate-specific membrane antigen after androgendeprivation therapy. Urology 48: 326-334.

28. Meller B, Bremmer F, Sahlmann CO, Hijazi S, Meller J, et al. (2015) Alterations in androgen deprivation enhanced prostate-specific membrane antigen (PSMA) expression in prostate cancer cells as a target for diagnostics and therapy. EJNMMI Res 5: 66.

29. Hope TA, Truillet C, Ehman EC, Aggarwal R, Ryan CJ, et al. (2017) 68GaPSMA-11 PET imaging of response to androgen receptor inhibition: first human experience. J Nucl Med 58: 81-84. 